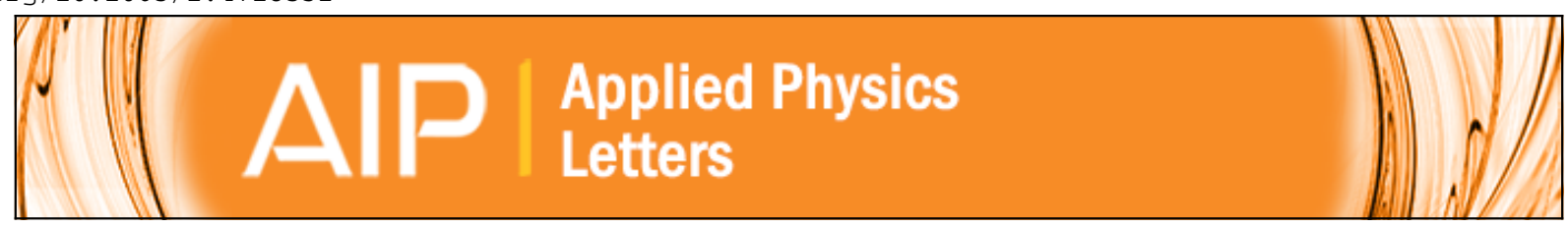

\title{
Shear-mode magnetostrictive/piezoelectric composite with an enhanced
}

\section{magnetoelectric coefficient}

Yaojin Wang, D. Hasanyan, Jiefang Li, D. Viehland, and Haosu Luo

Citation: Applied Physics Letters 100, 202903 (2012); doi: 10.1063/1.4718352

View online: http://dx.doi.org/10.1063/1.4718352

View Table of Contents: http://scitation.aip.org/content/aip/journal/apl/100/20?ver=pdfcov

Published by the AIP Publishing 


\title{
Shear-mode magnetostrictive/piezoelectric composite with an enhanced magnetoelectric coefficient
}

\author{
Yaojin Wang, ${ }^{1, a)}$ D. Hasanyan, ${ }^{1}$ Jiefang Li, ${ }^{1}$ D. Viehland, ${ }^{1}$ and Haosu Luo ${ }^{2}$ \\ ${ }^{1}$ Materials Science and Engineering, Virginia Tech, Blacksburg, Virginia 24061, USA \\ ${ }^{2}$ Shanghai Institute of Ceramics, Chinese Academy of Sciences, 215 Chengbei Road, Jiading, \\ Shanghai 201800, China
}

(Received 5 April 2012; accepted 29 April 2012; published online 15 May 2012)

\begin{abstract}
A magnetoelectric (ME) laminate heterostructure consisting of two shear-mode piezoelectric $\mathrm{Pb}\left(\mathrm{Mg}_{1 / 3} \mathrm{Nb}_{2 / 3}\right) \mathrm{O}_{3}-30 \mathrm{PbTiO}_{3}$ (PMN-PT) single crystal layers, a longitudinally magnetized magnetostrictive $\mathrm{Tb}_{0.3} \mathrm{Dy}_{0.7} \mathrm{Fe}_{1.92}$ alloy plate, and a mechanical clamping brass substrate has been demonstrated that has a notably superior ME effect relative to previous laminate configurations of these two materials. A giant ME coefficient of $7.5 \mathrm{~V} /(\mathrm{cm} \mathrm{Oe})$ at low frequencies under an optimal $\mathrm{dc}$ magnetic bias of $\sim 400$ Oe was found. The superior ME effects originate from the nature of heterostructure design, which allows the PMN-PT single crystals to operate in a shear mode that has maximum electro-mechanical coupling (i.e., $\mathrm{d}_{15}=6800 \mathrm{pC} / \mathrm{N}$ ). (C) 2012 American Institute of Physics. [http://dx.doi.org/10.1063/1.4718352]
\end{abstract}

Multiferroic materials with the coexistence of at least two ferroic orders (such as polarization, magnetization, and strain) have been of increasing interest both from fundamental and application perspectives. ${ }^{1,2}$ The coexistence of ferroelectricity and ferromagnetism, termed as magnetoelectric (ME) effect, ${ }^{1}$ is highly desireable. Although over ten different compound families have been widely investigated as multiferroic materials such as well-known $\mathrm{BiFeO}_{3}$ and rare earth magnanates, the ME effect in these single-phase materials are generally too weak to be technologically viable, especially at room temperature. ${ }^{2,3}$ Difficulties associated with uniting electric and magnetic ordering in a single phase have been circumvented by forming multi-phase multiferroic composites of piezoelectric and magnetostrictive components that can be electromagnetically coupled by stress mediation. ${ }^{3}$ The ME effect in these multi-phase composites is the result of a product tensor property of the magnetostrictive and piezoelectric components mechanically acting on each other. In this case, the ME effect depends on composite microstructure and configuration, coupling interaction across magneto-elasto-electric interfaces, and the individual properties of the composite components. ${ }^{2-12}$

To date, laminated composites of magnetostrictive Metglas or Terfenol-D and piezoelectric $\mathrm{Pb}\left(\mathrm{Mg}_{1 / 3} \mathrm{Nb}_{2 / 3}\right)$ $\mathrm{O}_{3}-30 \mathrm{PbTiO}_{3}$ (PMN-PT) or $\mathrm{Pb}(\mathrm{Zr}, \mathrm{Ti}) \mathrm{O}_{3}$ (PZT) possess the largest ME effects and highest sensitivity to magnetic field variations. This is due to the constituent phases having the highest individual magnetostrictive and piezoelectric coefficients. Using these component phases, various modes of operation have been studied, including: longitudinallongitudinal (L-L) ${ }^{8}$ longitudinal-transverse (L-T) ${ }^{7} \mathrm{TL}, \mathrm{TT}$, radial, push-pull, and bending ones. ${ }^{4}$ However, shear-mode PMN-PT single crystals have the highest piezoelectric coefficient values, as summarized in Table I. Thus, in principle, ME laminate composites using PMN-PT as the piezoelectric layer operated in a shear mode should provide the largest ME coefficients. However, this has not yet been found to be

${ }^{a)}$ Electronic mail: yaojin@vt.edu. the case. The challenge is to fabricate ME composites with materials having these key physical properties while achieving high shear-stress transfer between layers: otherwise the superior shear-mode properties of PMN-PT will be restricted. ${ }^{13}$

In this letter, we present the realization of a shear-mode laminate composite with high shear-stress transfer. The structure comprised a longitudinally magnetized Terfenol-D alloy plate, two shear-mode PMN-PT layers, and a mechanically clamping brass substrate. The shear mode ME coefficient is compared with that of L-L, L-T, and push-push mode ones. The results show that this shear mode configuration has a ME coefficient that is 2.5 times larger than that of other modes using ME laminates constructed of the same two phases and geometry. Furthermore, the observed giant ME coefficient in this heterostructure was identified to be due to shear extensional motions, rather than longitudinal extensional motions of PMN-PT layers.

Figure 1(a) shows a schematic diagram and the working principle of the proposed shear-mode ME laminate composite. The ME laminate consisted of two shear-mode PMN-PT layers, sandwiched between a longitudinally magnetized Terfenol-D plate and a mechanical clamping brass substrate. Single crystals of PMN-PT with dimensions of $10 \times 5 \times 0.5$ $\mathrm{mm}^{3}$ were grown in-house using a modified Bridgman technique, ${ }^{13}$ with their $\langle 111\rangle$ and $\langle 110\rangle$ crystallographic axes oriented in the length and thickness directions [see Fig. 1(b)]. After electroding with silver and poling along the length direction in a silicone oil bath at $120^{\circ} \mathrm{C}$, the silver electrodes were removed from the PMN-PT crystals. The de-electroded samples were then re-electroded along the thickness direction by sputtering gold at room temperature to avoid the occurrence of depolarization. The properties of the PMN-PT samples were then characterizated following the IEEE standard, as summarized in Table I. The Terfenol-D plate was commercially supplied (Gansu Tianxing Rare Earth Functional Materials Co., Ltd., China). They were of dimensions $40 \times 10 \times 1 \mathrm{~mm}^{3}$, with their long axes were directed along the [112] direction. These Terfenol-D layers were then 
TABLE I. Constituent material parameters of PMN-PT for transverse-extensional, longitudinal-extensional, and shear modes.

\begin{tabular}{|c|c|c|c|c|c|c|c|c|}
\hline \multirow[b]{2}{*}{ Parameter } & \multicolumn{3}{|c|}{ Piezoelectric constant $\left(10^{-12} \mathrm{pC} / \mathrm{N}\right)$} & \multicolumn{3}{|c|}{ Elastic compliance $\left(10^{-12} \mathrm{~m}^{2} / \mathrm{N}\right)$} & \multicolumn{2}{|c|}{ Dielectric constant } \\
\hline & $d_{31}$ & $d_{33}$ & $d_{15}$ & $s_{11}^{E}$ & $s_{12}^{E}$ & $s_{55}^{E}$ & $\varepsilon_{33}^{T} / \varepsilon_{0}$ & $\varepsilon_{11}^{T} / \varepsilon_{0}$ \\
\hline Transverse-extensional $^{\mathrm{a}}$ & -1883 & & & 112.0 & -31.1 & & 4033 & \\
\hline Longitudinal-extensional $^{\mathrm{b}}$ & & 2365 & & 41.2 & -17.3 & & 6833 & \\
\hline Shear-extensional $^{\mathrm{c}}$ & & & 6800 & 85.4 & & 212.2 & & 9540 \\
\hline
\end{tabular}

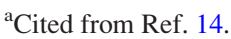

${ }^{\mathrm{b}}$ Cited from Ref. 15 .

${ }^{\mathrm{c}}$ Measured.

symmetrically stacked and bonded to PMN-PT ones using an epoxy resin (West System 106, USA). A brass substrate was bonded to the other side of the PMN-PT layers to form shear-mode three-phase ME laminate composites. In principle, the PMN-PT layers were operated in combined shear extensional and one-side longitudinal extensional modes, as illustrated in Figures 1(c) and 1(d). The Terfenol-D generated an elongation/shrinkage motion in response to an applied magnetic field $H$, while, a longitudinal-stress was applied to one-side of the PMN-PT plates and while the other side was clamped. Thus, the PMN-PT was excited into a one-side longitudinal extensional motion (due to the end parts of Terfenol-D) and a shear extensional motion (due to the center part of Terfenol-D).

First, the ME coefficient $\left(\alpha_{\mathrm{E}}\right)$ of the shear-mode heterostructure was measured for various dc magnetic biases $\left(H_{\mathrm{dc}}\right)$ in response to an ac magnetic field of $H_{\mathrm{ac}}=0.1$ Oe at a drive frequency of $f=1 \mathrm{kHz}$, as shown in Figure 2. The $H_{\mathrm{ac}}$ was provided by a Helmholtz coil driven by a lock-in amplifier (Stanford, SR-785). The $H_{\mathrm{dc}}$ was applied by a water-cooled, U-shaped electromagnet controlled by a dc power supplier/ amplifier (KEPCO, USA). The functional form of the variation of $\alpha_{\mathrm{E}}$ with $H_{\mathrm{dc}}$ was similar to that previously reported for magnetostrictive and piezoelectric composites. ${ }^{7-9}$ These

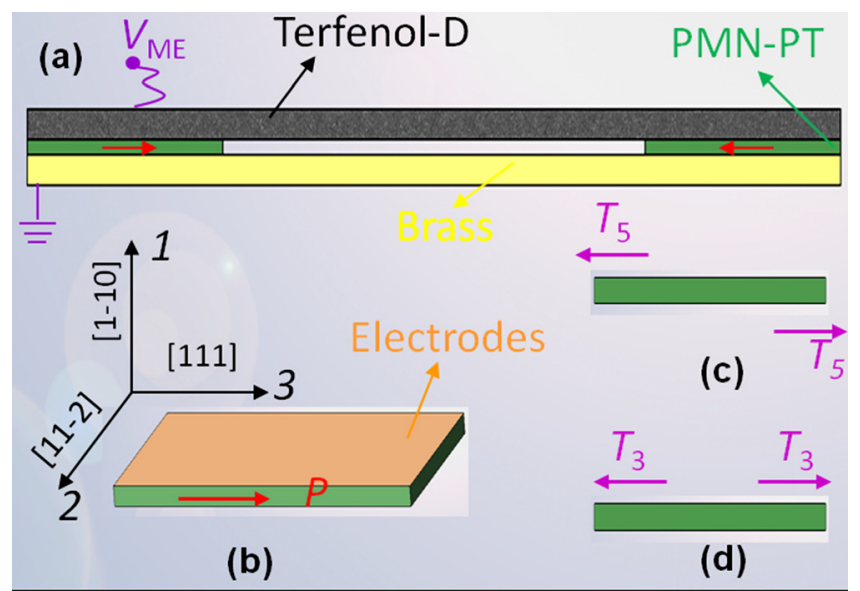

FIG. 1. (a) Schematic diagram of the proposed shear mode three-phase magnetostrictive/piezoelectric/brass heterostructure. (b) The coordinate system shows the crystalline directions of the PMN-PT single crystal. (c) and (d) illustrate the motion of the PMN-PT single crystal under shear extensional and longitudinal extensional stresses, respectively. The arrows $P$ and $T_{\mathrm{i}}(i=3$ and 5$)$ denote the polarization and stress directions. The Terfenol-D layer in the heterostructure has two functions: the end part (bonded to PMNPT) generates a longitudinal extensional stress $T_{3}$, and the center part (unbounded to PMN-PT) produces a shear extensional stress $T_{5}$. data reveal that the value of $\alpha_{\mathrm{E}}$ increased approximately linearly with increasing $H_{\mathrm{dc}}$ for low dc magnetic biases $\left(H_{\mathrm{dc}}<100 \mathrm{Oe}\right)$. A maximum value of $7.5 \mathrm{~V} /(\mathrm{cm} \mathrm{Oe})$ was observed at an optimal $H_{\mathrm{dc}} \sim 400 \mathrm{Oe}$, where values of $\alpha_{\mathrm{E}}$ for other mode composites were also maximized. ${ }^{7-9}$ It can be seen in Fig. 2 that the shear-mode $\alpha_{\mathrm{E}}$ exhibits a $\times 2.5, \times 22.1$, and $\times 3.5$ enhancement relative to $\mathrm{L}-\mathrm{T},{ }^{7} \mathrm{~L}-\mathrm{L},{ }^{8}$ and pushpull $^{9}$ modes, respectively.

As mentioned above, the PMN-PT layers in our laminate heterostructure were operated with combined shear and onesided longitudinal extensional motions. In order to separate the mechanism of the ME effect of the shear-mode laminate [see inset of Fig. 3(b)], an additional laminate composite that operated exclusively in a one-sided longitudinal mode was employed for comparative experiment. This pure one-sided longitudinal mode structure was made by a shear-mode PMN-PT sandwiched between a longitudinally magnetized Terfenol-D layer and an aluminum alloy clamping baseplate, as shown in the inset of Figure 3(c). The induced output ME voltage $V_{\mathrm{ME}}$ in the time-domain for these shear [see Fig. 3(b)] and one-sided longitudinal [see Fig. 3(c)] mode laminates were measured in response to $H_{\mathrm{ac} \text {,peak }}=1 \mathrm{Oe}$ at $f=1 \mathrm{kHz}$ [see Fig. 3(a)]. The results reveal that $V_{\mathrm{ME}}$ for the shear-mode laminate was much higher than that for the onesided longitudinal mode one (i.e., by a factor of $19.7 \times$ ).

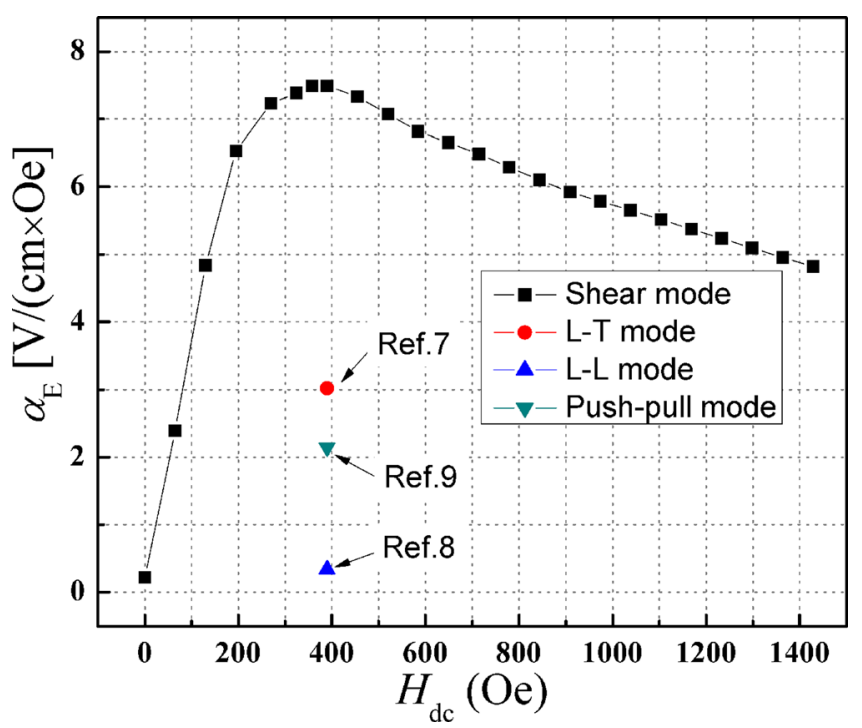

FIG. 2. ME coefficient $\alpha_{\mathrm{E}}$ as a function of dc magnetic bias $H_{\mathrm{dc}}$ for a shearmode Terfenol-D/PMN-PT laminate composite. The points denote the values of $\alpha_{\mathrm{E}}$ for the L-L mode, L-T mode, and push-pull mode Terfenol-D/ PMN-PT laminate composites (see Refs. 7-9). 


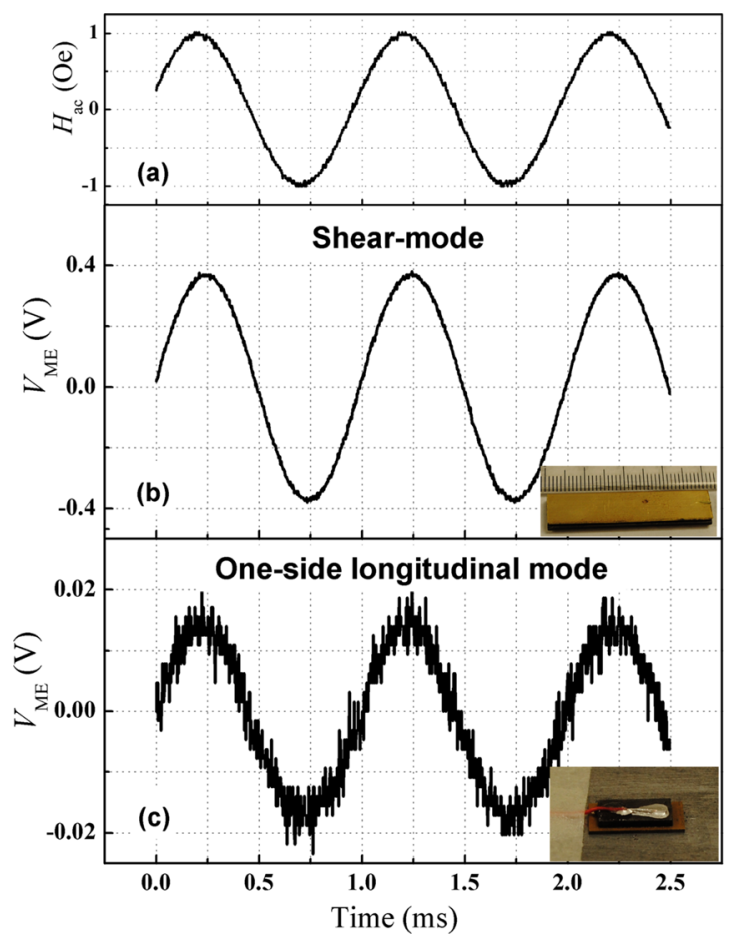

FIG. 3. Time-domain waveforms of (a) applied ac magnetic field $H_{\mathrm{ac}}$ and ME output voltage $V_{\mathrm{ME}}$ for (b) the shear mode Terfenol-D/PMN-PT laminates and (c) a one-sided longitudinal-extensional laminate. The inset shows the photos of the shear-mode and one-side longitudinal-mode laminate composites.

Clearly, the ME effect of the shear-mode laminate is dominated by the shear extensional motion, rather than the onesided longitudinal extensional one.

In summary, a shear-mode laminate composite consisting of a magnetostrictive alloy, piezoelectric single crystals, and a mechanical clamping substrate has been developed. In this shear-mode laminate, the PMN-PT layers operated with combined shear and one-sided longitudinal extensional motions. The dominate contribution to the ME coefficient was identified as a result of the shear extensional motion for the Terfenol-D/PMN-PT laminates. The higher elastoelectric coupling in this shear-mode laminate results in significantly enhanced $\mathrm{ME}$ coefficients relative to other operational modes-approximately $2.5 \times, 22.1 \times$, and $3.5 \times$ higher than prior L-T, L-L, and push-pull mode laminates, respectively.

This work was sponsored by the Office of Naval Research.

${ }^{1}$ N. A. Spaldin and M. Fiebig, Science 309, 391 (2005).

${ }^{2}$ J. Ma, J. Hu, Z. Li, and C.-W. Nan, Adv. Mater. 23, 1062 (2011).

${ }^{3}$ J. Jin, S.-G. Lu, C. Chanthad, Q. Zhang, M. A. Hague, and Q. Wang, Adv. Mater. 23, 3853 (2011).

${ }^{4}$ C. W. Nan, M. I. Bichurin, S. X. Dong, D. Viehland, and G. Srinivasan, J. Appl. Phys. 103, 031101 (2008).

${ }^{5}$ Y. J. Wang, D. Gray, D. Berry, J. Q. Gao, M. H. Li, J. F. Li, and D. Viehland, Adv. Mater. 23, 4111 (2011).

${ }^{6}$ G. Sreenivasulu, S. K. Mandal, S. Bandekar, V. M. Petrov, and G. Srinivasan, Phys. Rev. B 84, 144426 (2011).

${ }^{7}$ Y. J. Wang, S. W. Or, H. L. W. Chan, X. Y. Zhao, and H. S. Luo, J. Appl. Phys. 103, 124511 (2008).

${ }^{8}$ S. X. Dong, J. F. Li, and D. Viehland, Appl. Phys. Lett. 85, 5305 (2004).

${ }^{9}$ S. Dong, J. Zhai, F. Bai, J. F. Li, and D. Viehland, Appl. Phys. Lett. 87, 062502 (2005).

${ }^{10}$ Y. J. Chen, J. S. Gao, T. Fitchorov, Z. H. Cai, K. S. Ziemer, C. Vittoria, and V. G. Harris, Appl. Phys. Lett. 94, 082504 (2009).

${ }^{11}$ Y. Wang, D. Gray, J. Gao, D. Berry, M. Li, J. Li, D. Viehland, and H. Luo, J. Alloys Compd. 519, 1 (2012).

${ }^{12}$ Y. Wang, D. Gray, D. Berry, J. Gao, J. Li, D. Viehland, and H. Luo, Phys. Status Solidi (RRL) 5, 232 (2011).

${ }^{13}$ Y. J. Wang, S. W. Or, H. L. W. Chan, X. Y. Zhao, and H. S. Luo, Appl. Phys. Lett. 92, 123510 (2008).

${ }^{14}$ F. Wang, L. Luo, D. Zhou, X. Zhao, and H. Luo, Appl. Phys. Lett. 90, 212903 (2007).

${ }^{15}$ D. Zhou, F. Wang, L. Luo, J. Chen, W. Ge, X. Zhao, and H. Luo, J. Phys. D:Appl. Phys. 41, 185402 (2008). 